\section{Neue Aspekte zur Fries-Umlagerung ${ }^{[1][*]}$}

Von Franz Effenberger, Herbert Klenk und Peter Ludwig Reiter["]

Herrn Professor Karl Winnacker zum 70. Geburtstag gewidmet

Auch in den neuesten Arbeiten wird davon ausgegangen, daß die Fries-Umlagerung von im Kern nicht substituierten Phenylestern wie $(1), \mathrm{R}=\mathrm{R}^{\prime}=\mathrm{H}$, zu $o$ - und/oder $p$-Hydroxyaryl-ketonen (Acylphenolen) irreversibel ist ${ }^{[2]}$. Für 4Acyl-3-alkylphenole hatten Rosenmund und Schnurr ${ }^{[3]}$ sowie Miquel et al. ${ }^{14]}$ eine Umkehr der Fries-Umlagerung festgestellt, während Cullinane und $E d w a r d s^{[5]}$ behaupten, daß die Fries-Umlagerung auch in diesem Fall irreversibel ist.

Wir hatten früher gefunden, daß die Acylierung von Aromaten mit Carbonsäurechloriden oder -anhydriden und katalytischen Mengen Trifluormethansulfonsäure (TFMS) möglich ist ${ }^{[6]}$. Da der Kernacylierung von Phenolen große praktische Bedeutung zukommt, haben wir untersucht, ob die Fries-Umlagerung, die üblicherweise mit molaren Mengen $\mathrm{AlCl}_{3}$ durchgefüht wird, auch mit katalytischen Mengen TFMS möglich ist.

Erhitzt man in zugeschmolzenen Ampullen Lösungen von Phenylbenzoaten (1) in wasserfreiem Tetrachloräthan unter Zusatz von ca. $2 \mathrm{Mol} .-\%$ TFMS $24 \mathrm{~h}$ auf $170^{\circ} \mathrm{C}$, so werden neben den eingesetzten Estern (1) o-Hydroxyarylketone (2), Phenole (3) und Zersetzungsprodukte erhalten (Tabelle 1).<smiles>[R]c1cc([R])cc(OC(=O)c2ccccc2)c1</smiles>

(I)

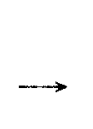<smiles>[R]c1cc(O)c(C(=O)OCc2ccccc2)c(CC)c1</smiles>

(2)

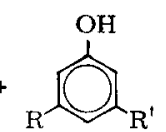

(3)
Tabelle 1. Fries-Umlagerung von Phenylbenzoaten (1) mit katalytischen Mengen Trifluormethansulfonsäure.

\begin{tabular}{|c|c|c|c|c|c|c|}
\hline \multirow{2}{*}{$\begin{array}{l}\text { Ausgangs- } \\
\text { material }\end{array}$} & \multirow[t]{2}{*}{$\mathbf{R}$} & \multirow[t]{2}{*}{$\mathbf{R}^{\prime}$} & \multirow[b]{2}{*}{ (l) } & \multicolumn{3}{|c|}{ Produkte $[\%][\mathrm{a}]$} \\
\hline & & & & (2) $[\mathrm{b}]$ & (3) & $\begin{array}{l}\text { Zers.- } \\
\text { Prod. }\end{array}$ \\
\hline (1a) & $\mathrm{H}$ & $\mathrm{H}$ & 48 & 39 & 5 & 8 \\
\hline$(1 b)$ & $\mathbf{H}$ & $\mathrm{NO}_{2}$ & 100 & - & - & - \\
\hline (1c) & $\mathbf{H}$ & $\mathrm{CH}_{3}$ & 40 & 49 & 11 & - \\
\hline$(1 d)$ & $\mathrm{H}$ & $\mathrm{CH}_{3} \mathrm{O}$ & 7 & 78 & - & 15 \\
\hline$(1 a)$ & $\mathrm{CH}_{3}$ & $\mathrm{CH}_{3}$ & 100 & - & - & - \\
\hline
\end{tabular}

[a] Ausbeuten aus mehreren Parallelversuchen gaschromatographisch ermittelt (Gaschromatograph Varian Serie 1200).

[b] $p$-Hydroxyaryl-ketone entstehen - wenn überhaupt - in Ausbeuten bis maximal $2 \%$

Bei präparativen Ansätzen (z. B. $0.1 \mathrm{~mol}(1 \mathrm{a}), 0.02 \mathrm{ml}$ TFMS in $100 \mathrm{ml}$ wasserfreiem Tetrachloräthan) sind die

[*] Prof. Dr. F. Effenberger, Dipl.-Chem. H. Klenk und Dipl.-Chem. P. L. Reiter Institut für Organische Chemie der Universität 7 Stuttgart, Pfaffenwaldring 55

[**] Diese Arbeit wurde von der Deutschen Forschungsgemeinschaft und dem Fonds der Chemischen Industrie unterstiutzt.
Ausbeuten innerhalb der Fehlergrenzen mit denen in Tabelle 1 vergleichbar. Der Fries-Verschiebung mit TFMS unterliegen auch die Phenylester aliphatischer Carbonsäuren.

Die Ergebnisse zeigen, daß besonders Elektronendonoren an C-3 des Phenols die TFMS-katalysierte Umlagerung begünstigen: aus ( $1 d$ ) erhielten wir in 78-proz. Ausbeute $(2 d)$, während $(1 b)$ unverändert bleibt. Es war deshalb überraschend, daß ( 1 e) nicht umlagert wird, obwohl die Methylgruppen an C-3 und C-5 eine Kernacylierung begünstigen sollten. Eine Erklärung für diesen Befund sehen wir in der Reversibilität der Fries-Umlagerung.

Zur Prüfung dieser Hypothese haben wir einige o-Hydroxyaryl-ketone (2) unter den oben angegebenen Bedingungen mit TFMS erhitzt. Die Ergebnisse dieser Versuche bestätigen die Annahme der Reversibilität der Fries-Umlagerung (Tabelle 2)

Tabelle 2. Retro-Fries-Umlagerung von o-Hydroxyaryl-ketonen (2) mit katalytischen Mengen Trifluormethansulfonsäure.

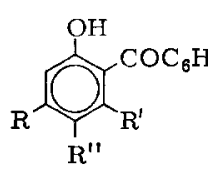

(2)

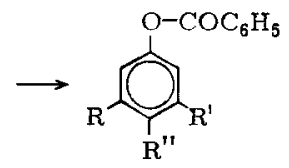

(I)

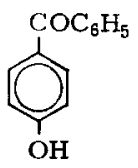

(4)

\begin{tabular}{llllllll}
\hline $\begin{array}{l}\text { Ausgangs- } \\
\text { material }\end{array}$ & $\mathbf{R}$ & $\mathbf{R}^{\prime}$ & $\mathbf{R}^{\prime \prime}$ & & \multicolumn{2}{c}{ Produkte [\%] [a] } \\
& & & & $(2)$ & $(1)$ & $\begin{array}{l}\text { Zers. } \\
\text { Prod. }\end{array}$ \\
\hline$(2 a)$ & $\mathrm{H}$ & $\mathrm{H}$ & $\mathrm{H}$ & 75 & 25 & - \\
$(2 a)$ & $\mathrm{CH}_{3}$ & $\mathrm{CH}_{3}$ & $\mathrm{H}$ & - & 90 & 10 \\
$(2 f)$ & $\mathrm{H}$ & $\mathbf{H}$ & $\mathrm{CH}_{3}$ & 47 & 53 & - \\
$(4)$ & & & & $(4), 23$ & $(1 a), 40$ & 28 \\
& & & & $(2 a), 9$ & & \\
\hline
\end{tabular}

[a] Vgl. Fußnote [a] in Tabelle 1.

Die Produktverteilung bei der Fries- und Retro-Fries-Umlagerung (Tabelle 1 bzw. 2) ist qualitativ zwar vergleichbar, genügt jedoch nicht den Kriterien eines reversibel einstellbaren Gleichgewichts, so daß weitere Arbeiten notwendig sind.

Die bisherigen Ansichten zum Mechanismus der FriesUmlagerung müssen nach den vorliegenden Ergebnissen, die die Reversibilität dieser Umlagerung beweisen, angezweifelt werden, zumal wir auch bei Verwendung typischer Friedel-Crafts-Katalysatoren wie Eisen(II)-chlorid RetroFries-Umlagerung gefunden haben.

Aus den bisherigen Ergebnissen schließen wir, daß die Produktbildung bei der säurekatalysierten Fries-Umlagerung in vielen Fällen thermodynamisch kontrolliert ist. Phenylester und Hydroxyaryl-ketone sind offensichtlich von vergleichbarer Stabilität und liegen deshalb nebeneinander vor. Bei einer sterischen Hinderung wie in (2e) verschiebt sich die Produktverteilung dagegen zugunsten des sterisch ungehinderten Esters (Tabelle 2).

[1] Zur elektrophilen Aromatensubstitution, 8. Mitteilung. - 7. Mitteilung: $F$. Effenberger u. H. Klenk, Chem. Ber., im Druck.

[2] M. J.S. Dewar u. L. S. Hart, Tetrahedron 26, $973(1970)$

[3] K. W. Rosenmund u. W. Schnurr, Liebigs Ann. Chem. 460, 56 (1928)

[4] J. F. Miquel, P. Miller u. Nh. Ph. But-Hoi, Bull. Soc. Chim. Fr. 1965,633

[5] N. M. Cullinane u. B. F. R. Edwards, J. Chem. Soc. 1958, 434. [6] F. Effenberger u. G. Epple, Angew. Chem. 84, 295 (1972); Angew. Chem. internat. Edit. 11, 300 (1972). 
Die praktisch ausschließliche ortho-Umlagerung stützt ebenfalls die Annahme der thermodynamischen Kontrolle, da bei $o$ - im Gegensatz zu p-Hydroxyaryl-ketonen eine intramolekulare Wasserstoffbrücken-Stabilisierung anzunehmen ist.

Eingegangen am 24. Juli $1973 \quad$ [Z 882] 